\title{
Effective Atomic Number Determination of Rare Earth Oxides with Scattering Intensity Ratio
}

\author{
A. Turşucu, ${ }^{1}$ D. Demir, ${ }^{2}$ and P. Önder ${ }^{2}$ \\ ${ }^{1}$ Department of Energy System Engineering, Faculty of Engineering, Sirnak University, 73000 Şırnak, Turkey \\ ${ }^{2}$ Department of Physics, Faculty of Sciences, Ataturk University, 25240 Erzurum, Turkey
}

Correspondence should be addressed to A. Turşucu; ahmettursucu@sirnak.edu.tr

Received 9 July 2013; Accepted 3 October 2013

Academic Editor: Borut Mavko

Copyright ( 2013 A. Turşucu et al. This is an open access article distributed under the Creative Commons Attribution License, which permits unrestricted use, distribution, and reproduction in any medium, provided the original work is properly cited.

\begin{abstract}
Effective atomic numbers $\left(Z_{\text {eff }}\right)$ of scientific samples (rare earth) were determined experimentally by scattering of $59.54 \mathrm{keV}$ gamma photons from $5 \mathrm{Ci}^{241} \mathrm{Am}$ annular radioactive source. The scattered gamma photons were collected by using a high-resolution $\mathrm{HPGe}$ semiconductor detector placed at $167^{\circ}$ to the incident beam. This experiment was carried out on several elements in the atomic range $4 \leq Z \leq 82$ for $59.54 \mathrm{keV}$ incident photons. Photopeak efficiency and air and sample absorption corrections were performed on Rayleigh to Compton scattering intensity ratio; then the ratio was plotted as a function of atomic number and a fit curve was constituted. The effective atomic numbers of rare earth oxide samples were determined by this fit curve. Also, related parameters were determined by absorption technique with the same incident photon energy. Obtained values from this fit curve were compared to theoretical values and were found to closely agree with theoretical calculations.
\end{abstract}

\section{Introduction}

Effective atomic number is an important parameter that should be determined for materials in composite, alloy, and mixture form. Reliable data on the effective atomic numbers were used in radiation biology, medical physics, radiation dosimetry, nuclear industry, and space research programs. Also, the effective atomic number is used for determining the $\mathrm{X}$ - and $\gamma$-ray absorption fraction of materials. There are a few techniques (particle induced X-ray emissions (PIXE), proton induced gamma ray emission (PIGE), fast neutron activation analysis (FNNA), induced coupled plasma (ICP), electrical impedance methods, XRF, chemical analysis, and atomic absorption spectroscopy) to define the effective atomic number of composite materials that was created by two or more constituents. One of these techniques is total photon interaction calculation by using transmission experiments. In addition to these techniques, intensity ratio of scattered photons, emerging from suitable sample-source and detector arrangement, was a significant experimental method to determine effective atomic number in a nondestructive way. The scattered intensity ratio method is regardless of density of the sample and depends on the mixture of composite material. The above-mentioned research techniques used in a present study are subapplication of the XRF technique.

Rare earth oxides of lanthanids are widely used in scientific, industrial, commercial, and medical applications. For example, praseodymium oxide in solid solution with ceria or ceria-zirconia is used as oxidation catalysis; europium oxide is used in nuclear reactor as a neutron absorber, fluorescent lamps, and screening for Down syndrome and other genetic diseases. In addition to these applications, terbium oxide (green phosphors in fluorescent lamps and color TV tubes) and dysprosium oxide (neutron-absorbing control rods in nuclear reactors, various data storage applications, and in improving the corrosion resistance of the magnets) are also important.

In recent years determination of the effective atomic number is increasingly studied. Due to the advantages, most of the investigations on this subject have been carried out using scattering intensity ratios. Singh et al. [1] have measured the effective atomic numbers of scientific (lanthanide oxides and alloys of lead and tin of known composition) and biological samples (iodine content of tissues) using scattering 
intensity ratio of $145 \mathrm{keV}$ gamma rays. Singh et al. [2] have determined the effective atomic number of scientific samples by scattering intensity ratio of $59.54 \mathrm{keV}$ gamma photons. There are some points should be considered in the determination process of the effective atomic number by scattering intensity ratio method. One of these points is thickness of sample. Increasing thicknesses are creating multiple scattering centers on the target. Therefore, the multiple scattering densities are increasing with the increasing thickness. Singh et al. [3] have determined the effect of multiple scattering on the effective atomic number. The effective atomic number of composite materials using Rayleigh to Compton scattering of $279 \mathrm{keV}$ gamma rays was investigated by Singh et al. [4].

In this paper, we have determined the effective atomic number of rare-earth samples by scattering intensity ratio method. For this purpose, $\mathrm{La}_{2} \mathrm{O}_{3}, \mathrm{Pr}_{2} \mathrm{O}_{3}, \mathrm{Eu}_{2} \mathrm{O}_{3}, \mathrm{Sm}_{2} \mathrm{O}_{3}$, and $\mathrm{Er}_{2} \mathrm{O}_{3}$ rare earth samples were investigated in $167^{\circ}$ scattering angle with HPGe semiconductor detector.

\section{Theory}

When an X-ray impinges upon a sample, three things may happen: the incident X-ray may be diffracted, scattered, or absorbed. Scattering will occur when an X-ray photon collides with one of the electrons of the absorbing element. Where this collision is elastic, that is, when no energy is lost in the collision process, the scattering is said to be coherent (Rayleigh) scattering. Since no energy change is involved, the coherently scattered radiation will retain exactly the same wavelength as the incident beam. The probability of Rayleigh scattering occurrence theoretically is given as

$$
\frac{d \sigma_{R}}{d \Omega}=\frac{r_{0}^{2}}{2}\left(1+\cos ^{2} \theta\right)|F(q, Z)|^{2},
$$

where $F(q, Z)$ is the atomic form factor, $q$ representing the momentum transferred to the electron. $r_{0}(=2.8179 \times$ $10^{-15} \mathrm{~m}$ ) is the classical electron radius and $\theta$ is the scattering angle.

Compton scattering is the interaction of a high energy photon with an electron and the resulting "scattered" photon which has a reduced frequency and therefore reduced energy. Compton scattering requires that light is viewed as a particle and not just a wave because it is the "collision" of the photon with the electron and the exchange of energy, which accounts for the shift in energy. The occurrence probability of Compton scattering is provided by Klein-Nishina relation:

$$
\left(\frac{d \sigma}{d \Omega}\right)_{\mathrm{KN}}=\frac{1}{2} r_{0}^{2}\left(\frac{E}{E_{0}}\right)^{2}\left(\frac{E_{0}}{E}+\frac{E}{E_{0}}-\sin ^{2} \theta\right),
$$

where $E$ and $E_{0}$ are scattered and incident photon energies. In large values of the momentum transfer, incoherent scattering function $(S(q, Z))$ is equal to number of the atomic electrons, and when momentum transfer approaches the zero, $S(q, Z)$ is approaching the zero at the same time. In this instance, the ratio of Rayleigh scattering cross-section to Compton scattering cross-section is given as

$$
R=\frac{(d \sigma / d \Omega)_{R}}{(d \sigma / d \Omega)_{\mathrm{aC}}} \propto \frac{|F(q, Z)|^{2}}{S(q, Z)},
$$

where $(d \sigma / d \Omega)_{\mathrm{aC}}$ is the atomic Compton scattering crosssection that occurs from scattering by bound electrons. The atomic Compton scattering cross-section is given as

$$
\left(\frac{d \sigma}{d \Omega}\right)_{\mathrm{aC}}=S(q, Z)\left(\frac{d \sigma}{d \Omega}\right)_{\mathrm{KN}} .
$$

Equation (3) represents the ratio of Rayleigh to Compton scattering. The $R$ value obtained from this equation is proportional to the atomic number of investigated material. This proportion depends on $F^{2} / S$ ratio. Theoretical data for calculation of the Rayleigh to Compton cross-section from parameters for $F(q, Z)$ and $S(q, Z)$ was created by Hubbell et al. [5].

The $R$ value, which is directly related to the atomic number of elemental interest, is calculated via weighting of the atomic percentages of elements using atomic form factor and incoherent scattering function:

$$
R=\frac{(d \sigma / d \Omega)_{R}}{(d \sigma / d \Omega)_{\mathrm{aC}}} \propto \frac{\sum_{j=1}^{n} a_{j}^{\mathrm{at}}\left|F\left(q, Z_{j}\right)\right|^{2}}{\sum_{j=1}^{n} a_{j}^{\mathrm{at}} S\left(q, Z_{j}\right)} ;
$$

here $a_{j}^{\text {at }}$ represents the atomic percentages and is calculated by $W_{j}$ and the atomic mass $A_{j}$ of the $j$ th element as

$$
a_{j}^{\text {at }}=\frac{\left(W_{j} / A_{j}\right)}{\sum_{j}\left(W_{j} / A_{j}\right)} \text {. }
$$

The effective atomic number of material is calculated via the ratio of its atomic cross-section to electronic cross-section:

$$
Z_{e}=\frac{\sigma_{t}}{\sigma_{e}}
$$

The atomic cross-section $\left(\sigma_{t a}\right)$ is calculated as

$$
\sigma_{t a}=\frac{\mu_{m} N}{N_{A}}
$$

where $\mu_{m}, N$, and $N_{A}$ represent mass attenuation coefficient, Avogadro's number, and atomic weight, respectively [6]. The total electronic cross-section for interest element is given as follows:

$$
\sigma_{t, e}=\frac{1}{N} \sum_{j} \frac{a_{j} A_{j}}{Z_{j}}\left(\frac{\mu}{\rho}\right)
$$

here $a_{j}$ is the number of atoms of element $j$ relative to the total number of atoms of all elements in the mixture. $Z_{j}$ is the atomic number of the $j$ th element in a molecule, and $(\mu / \rho)_{j}$ is the total mass attenuation coefficient of the $j$ th element in a molecule [4]. 


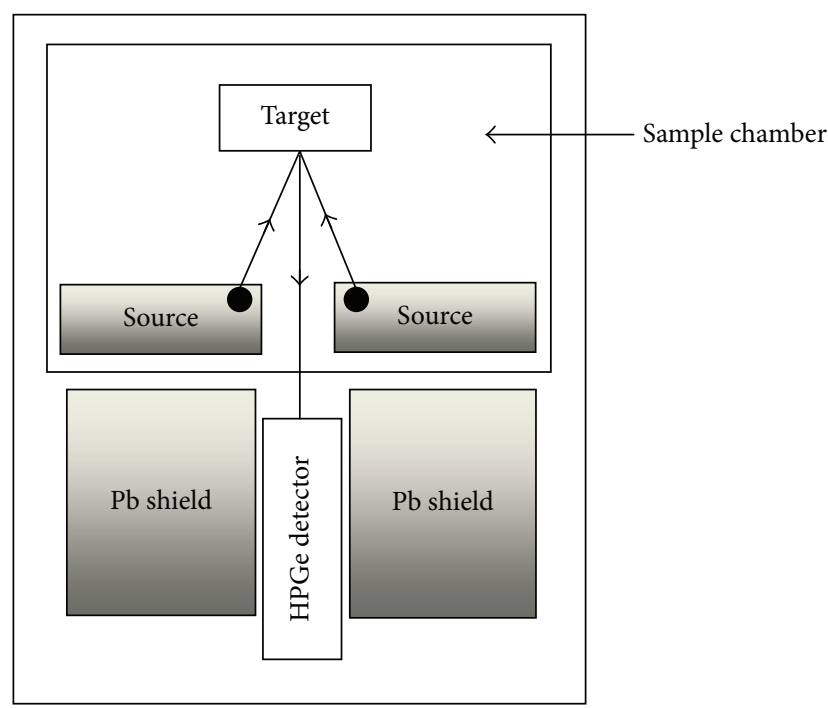

FIGURE 1: Experimental setup.

\section{Experimental Setup and Present Measurements}

In this study, firstly we were aimed to determine the scattering intensity ratios of scientific samples to obtain their effective atomic numbers. For this purpose, we used $5 \mathrm{Ci}{ }^{241} \mathrm{Am}$ annular source as an exciter and HPGe semiconductor detector to collect the scattering photons. The HPGe detector was a DSG planar high purity germanium crystal with a diameter of $16 \mathrm{~mm}$, a length of $10 \mathrm{~mm}$, a beryllium window of $0.12 \mathrm{~mm}$, and active area of $200 \mathrm{~mm}^{2}$. The schematic arrangement of the experimental setup used in the present study is shown in Figures 1 and 2. A bias voltage of $-1500 \mathrm{~V}$ was applied to the detector with a resolution of $182 \mathrm{eV}$ at $5.9 \mathrm{keV}$. The rareearth samples of thickness ranging from 0,189 to $0,265 \mathrm{~g} / \mathrm{cm}^{2}$ were used. In this study, the study samples were $\mathrm{La}_{2} \mathrm{O}_{3}$, $\mathrm{Pr}_{2} \mathrm{O}_{3}, \mathrm{Eu}_{2} \mathrm{O}_{3}, \mathrm{Sm}_{2} \mathrm{O}_{3}$, and $\mathrm{Er}_{2} \mathrm{O}_{3}$. Spectrum of samples was processed by using a Canberra (AccuSpec) Pc-based multichannel analyzer card. For the best counting rates from detector we set the time constant of Ortec model 472 amplifier at $6 \mu \mathrm{s}$. Operating parameters of the system were governed and controlled by the computer program Genie-2000. The data were collected into 1024 channels of the MCA. Data were analyzed by the Origin 7.5 software program. A typical spectrum of $\mathrm{La}_{2} \mathrm{O}_{3}$ target is shown in Figure 3. Also a typical spectrum of $\mathrm{V}$ (pure vanadium) target is shown in Figure 4.

\section{Result and Discussion}

This experiment represents a procedure to determine effective atomic number of rare earth through measurements of Rayleigh and Compton scattering intensity using photon energy of $59.54 \mathrm{keV}$. The $Z_{\text {eff }}$ values of rare earth were needed to be determined precisely because of their applications especially in space physics, nuclear physics, medical physics, radioisotope monitoring, neutron capturing, lasers, scattering and attenuation of radiation, testing of multicomponent,

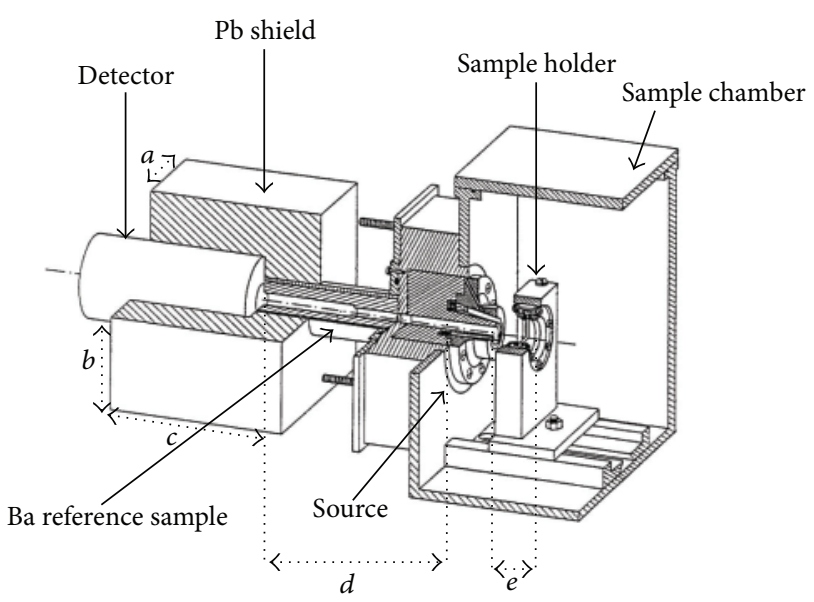

Figure 2: Sample chamber $(a=6.5 \mathrm{~cm}, b=6.3 \mathrm{~cm}, c=13.5 \mathrm{~cm}, d=$ $11 \mathrm{~cm}, e=5 \mathrm{~cm})$.

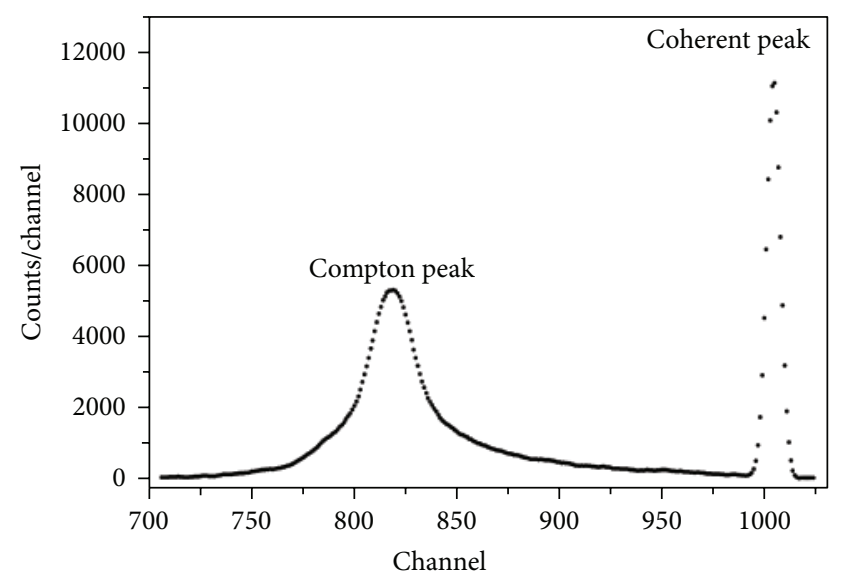

Figure 3: Typical observed spectra at $167^{\circ}$ from $\mathrm{La}_{2} \mathrm{O}_{3}$ target when irradiated by $59.54 \mathrm{keV}$ incident photon energy.

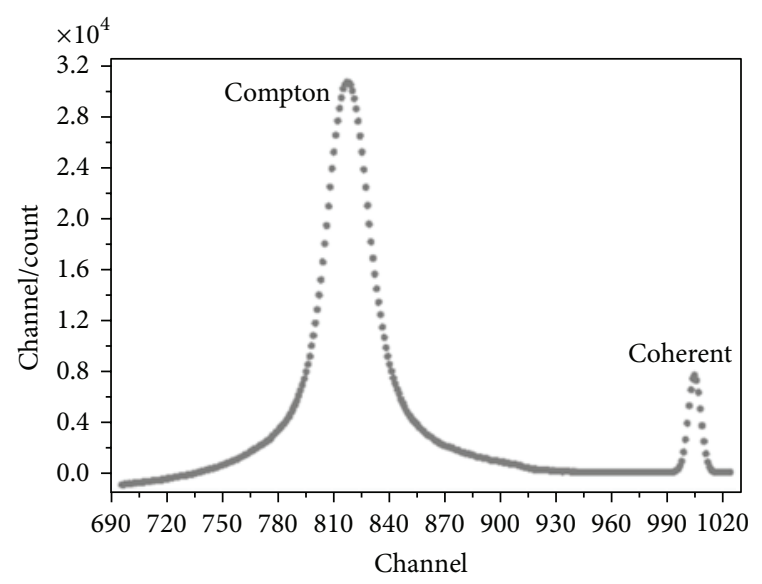

FIgure 4: A typical Compton and coherent scattering spectrum from pure Vanadium Target. 
designs of radiation shielding, calculations of absorbed dose in radiotherapy, and many other radioactive applications.

In the present measurements, a target of known atomic number (Be, C, Al, Si, Ca, Ti, V, Fe, Co, Ni, Cu, Zn, Y, Zr, $\mathrm{Nb}, \mathrm{Mo}, \mathrm{Ru}, \mathrm{Rh}, \mathrm{Pd}, \mathrm{Ag}, \mathrm{Cd}, \mathrm{Sn}, \mathrm{La}, \mathrm{Dy}, \mathrm{Yb}, \mathrm{Ta}, \mathrm{Au}$, and Pb) and thickness $\left(0.022-0.865 \mathrm{~g} / \mathrm{cm}^{2}\right)$ was used. The scattered spectra of the targets are recorded for a period of 3600$61200 \mathrm{~s}$. The background was recorded after removing the target out of the primary beam to permit the registration of events due to cosmic rays and to any other process independent of the target. The background count rate was subtracted from measurements. In the recorded scattered spectra, Rayleigh and Compton peaks were not simple. They were with superimposed continuum; hence, the following formula was used to subtract the additional unwanted counts included in this process:

$$
\text { Peak area }=\sum_{i=A}^{B} C_{i}-(B-A) \frac{C_{A}+C_{B}}{2},
$$

where $A$ and $B$ are the channel numbers specifying the region of observed Photopeak, $C_{A}$ and $C_{B}$ are the counts at the respective channels, and $C_{i}$ is the simple integration of counts between the limits $A-B$. These observed intensities were corrected for Photopeak efficiency of the HPGe detector, absorption in the air between target and the detector, and selfabsorption in the primary target, as per the relation

$$
N_{\text {actual }}=\frac{N_{\text {obs }}}{\varepsilon_{\gamma} \beta_{\gamma a} \beta_{\gamma t}}
$$

here $N_{\text {obs }}$ is the observed intensity under the Rayleigh (or Compton) peak; $\beta_{\gamma a}$ is the correction factor for absorption of photons in the air present between the target and the detector; $\beta_{\gamma t}$ is the self-absorption correction factor for the scattered photons in the target; $\varepsilon_{\gamma}$ is the Photopeak efficiency of the detector for Rayleigh (or Compton) scattered photons. The self-absorption correction factor was determined using the work of Weyrich [7]:

$$
\beta=\frac{1-\exp \left[-\left(\mu_{1}+\mu_{2}\right) d\right]}{\left(\mu_{1}+\mu_{2}\right) d}
$$

here $\mu_{1}$ and $\mu_{2}$ represent the absorption coefficients of incident and scattered photons, respectively, and $d$ is the sample thickness.

The Photopeak efficiency curve for the HPGe detector is shown in Figure 5 and created experimentally 13.81$1212.95 \mathrm{keV}$ photon energy by using $\mathrm{Am}^{241}, \mathrm{Ba}^{133}, \mathrm{Cs}^{137}$, and $\mathrm{Eu}^{152}$. Each of these radioactive sources of known activity was placed at the position of the elemental target, and the energy spectra were recorded by the HPGe detector. The solid angle subtended by the detector at the centre of the source is thus the same as in the actual measurements. For a gamma emitted from a radioisotope source with a known activity, the efficiency is given by the expression

$$
\varepsilon_{\gamma}=\frac{A_{c}}{N_{0} p_{\gamma} e^{-\lambda t}}
$$

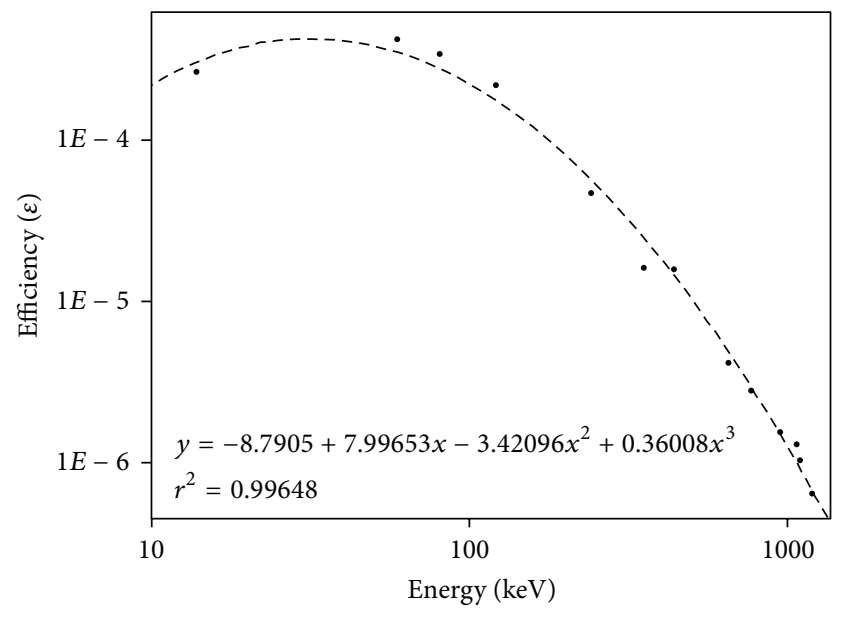

FIGURE 5: The photopeak efficiency curve for HPGe detector.

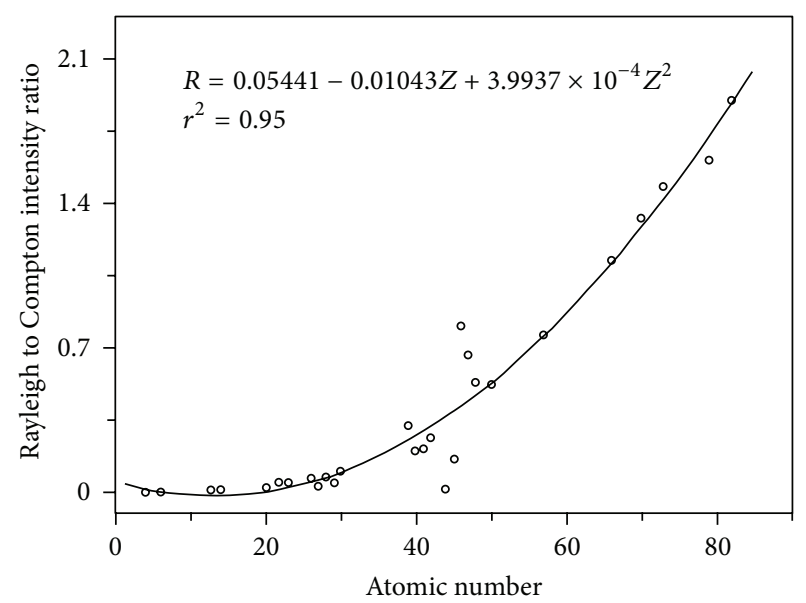

FIGURE 6: Rayleigh to Compton scattered intensity ratio as a function of atomic number.

where $A_{c}$ is net peak area in counts, $N_{0}$ is the activity of source at the time of standardization, $p_{\gamma}$ is the absolute gamma ray emission probability, $\lambda$ is the decay constant, and $t$ is the elapsed time since standardization. The energies and emission probabilities of the sources used in the present work were taken from Table of Radioactive Isotopes [8].

The intensity ratio of Rayleigh and Compton scattered peaks for different targets of known atomic number is plotted as a function of atomic number and is given in Figure 6. In this figure, due to lack of the same features (size, thickness) of the target samples, some deviations occurred in the atomic range $40 \leq Z \leq 50$. The solid curve represents the best-fit curve through experimental data points corresponding to the intensity ratio of Rayleigh to Compton scattering in Figure 6 [9]. The equation for the best-fit curve is

$$
R=0.05441-0.01043 Z+3.9937 \times 10^{-4} Z^{2} .
$$

Each of the rare earth samples is replaced with the elemental target and the scattered spectra are recorded. The intensities under the observed Rayleigh and Compton peaks were evaluated. The use of (11) provides the actual 
TABLE 1: Rayleigh to Compton scattered intensity ratio and experimental and theoretical effective atomic numbers of rare earth oxides at $59.54 \mathrm{keV}$.

\begin{tabular}{|c|c|c|c|c|}
\hline Rare earth & Rayleigh to Compton intensity ratio & Experimental effective atomic number & Other experimental & Theoretical \\
\hline $\mathrm{La}_{2} \mathrm{O}_{3}$ & $0.535 \pm 0.002$ & $50.14 \pm 0.76$ & - & 55.79 \\
\hline $\mathrm{Pr}_{2} \mathrm{O}_{3}$ & $0.801 \pm 0.002$ & $58.22 \pm 0.74$ & $-55.11^{*}$ & 57.85 \\
\hline $\mathrm{Sm}_{2} \mathrm{O}_{3}$ & $0.978 \pm 0.002$ & $62.89 \pm 0.81$ & - & 60.91 \\
\hline $\mathrm{Eu}_{2} \mathrm{O}_{3}$ & $1.04 \pm 0.002$ & $65.15 \pm 0.94$ & $-58.88^{*}$ & 61.89 \\
\hline $\mathrm{Er}_{2} \mathrm{O}_{3}$ & $1.21 \pm 0.003$ & $68.41 \pm 1.22$ & - & 67.21 \\
\hline
\end{tabular}

${ }^{*}$ Singh et al. [1].

intensities under these peaks originating from interactions of $59.54 \mathrm{keV}$ photons with the target atoms (electrons) and the scattered photons traveling in direction of the detector. The effective atomic number of $\mathrm{La}_{2} \mathrm{O}_{3}, \mathrm{Pr}_{2} \mathrm{O}_{3}, \mathrm{Eu}_{2} \mathrm{O}_{3}, \mathrm{Sm}_{2} \mathrm{O}_{3}$, and $\mathrm{Er}_{2} \mathrm{O}_{3}$ is then deduced from the best-fit curve of Figure 6 . Also, the effective atomic numbers of these samples were theoretically evaluated from known elemental concentration of the constituent elements using mixture rule and WinXCom program [10]. The present effective atomic numbers, other experimental values in the literature, and Rayleigh to Compton scattered intensity ratios are given in Table 1. Also theoretical values of the effective atomic numbers of rare earth are given in Table 1. The overall error in the experimental parameters was the sum of the uncertainties in different factors, namely, the evaluation of peak areas (1.69$3.71 \%)$, target mass thickness (1.45-3.20\%), the Photopeak efficiency (1.10-2.04\%), and statistical error $(<1.00 \%)$. Total errors affecting the experimental parameters were calculated between 2.68 and 5.40\%. Theoretical calculations do not take into account molecular and solid-state effects. XCOM database neglects a strong interaction between atoms and Coulomb interaction between the photoelectron and the positively charged ion. Also, database does not calculate energy absorption coefficients. This situation can be a reason to discrepancy between theoretical and experimental values.

It was clearly seen that the experimental measured values were in good agreement with calculated theoretical values. Also, the present measurements showed the usability of this experimental technique to measure effective atomic number. To obtain more definite conclusions on the effective atomic number using this method, more experimental data are clearly needed, particularly for different scattering angles and chemical compounds.

\section{Conclusion}

There are many articles about determining the effective atomic number of composite materials. Some of these articles are related with mass attenuation technique. We have presented the effective atomic number of some rare earth with scattering intensity ratio. For this purpose, $\mathrm{La}_{2} \mathrm{O}_{3}, \mathrm{Pr}_{2} \mathrm{O}_{3}$, $\mathrm{Eu}_{2} \mathrm{O}_{3}, \mathrm{Sm}_{2} \mathrm{O}_{3}$, and $\mathrm{Er}_{2} \mathrm{O}_{3}$ rare earth samples were investigated in $167^{\circ}$ scattering angle with HPGe semiconductor detector. Our calculations include sample, air attenuation correction and detectors photopeak efficiency.

\section{Highlights}

(i) The effective atomic number of composite material is an important parameter.

(ii) Scattering intensity ratio is a significant technique to determine effective atomic numbers.

(iii) Our present experimental values in good agreement with theory and other experimental.

\section{Conflict of Interests}

The authors declare that there is no conflict of interests regarding the publication of this paper.

\section{References}

[1] M. P. Singh, A. Sharma, B. Singh, and B. S. Sandhu, "Nondestructive evaluation of scientific and biological samples by scattering of $145 \mathrm{keV}$ gamma rays," Radiation Measurements, vol. 45, no. 8, pp. 960-965, 2010.

[2] M. P. Singh, A. Sharma, B. Singh, and B. S. Sandhu, "A nondestructive technique for assigning effective atomic number to scientific samples by scattering of $59.54 \mathrm{keV}$ gamma photons," Nuclear Instruments and Methods in Physics Research A, vol. 619, no. 1-3, pp. 63-66, 2010.

[3] M. Singh, B. Singh, and B. S. Sandhu, "Investigations of multiple scattering of $320 \mathrm{keV} \gamma$ rays: a new technique for assigning effective atomic number to composite material," Physica Scripta, vol. 79, no. 3, Article ID 035101, 8 pages, 2009.

[4] M. P. Singh, B. S. Sandhu, and B. Singh, "Measurement of the effective atomic number of composite materials using Rayleigh to Compton scattering of $279 \mathrm{keV}$ gamma rays," Physica Scripta, vol. 76, no. 4, pp. 281-286, 2007.

[5] J. H. Hubbell, E. H. Veigele, R. T. Briggs, D. T. Brown, R. J. Cromer, and J. Howertan, "Atomic form factors, Incoherent scattering functions and photon scattering cross sections," Journal of Physical and Chemical Reference Data, vol. 4, pp. 471538, 1975.

[6] D. C. Wang, P. Luo, and H. Yang, "Measurement of the mass attenuation coefficients for $\mathrm{SiH}_{4}$ and $\mathrm{Si}$," Nuclear Instruments and Methods in Physics Research B, vol. 95, no. 2, pp. 161-165, 1995.

[7] W. Weyrich, "The electron momentum distribution in solid potassium fluoride, studied by compton scattering," Berichte der Bunsengesellschaft für Physikalische Chemie, vol. 79, no. 11, pp. 1085-1095, 1975. 
[8] R. B. Firestone and L. P. Ekström, WWW Table of Radioactive Isotopes, version 2.1, January 2004, http://ie.lbl.gov/toi/.

[9] D. Demir and A. Turşucu, "Measurement of the effective atomic number of $\mathrm{Fe}_{x} \mathrm{Cr}_{1-x}$ and $\mathrm{Fe}_{x} \mathrm{Ni}_{x}$ alloys using scattering of gamma rays," Journal of Alloys and Compounds, vol. 581, pp. 213216, 2013.

[10] L. Gerward, N. Guilbert, K. B. Jensen, and H. Levring, "X-ray absorption in matter. Reengineering XCOM," Radiation Physics and Chemistry, vol. 60, no. 1-2, pp. 23-24, 2001. 


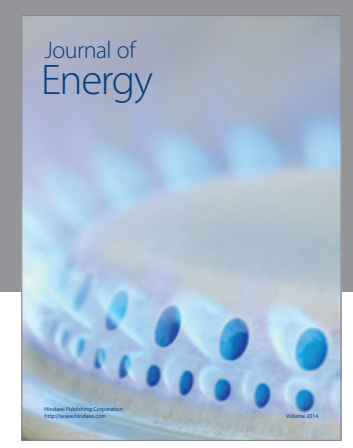

Journal of

Industrial Engineering
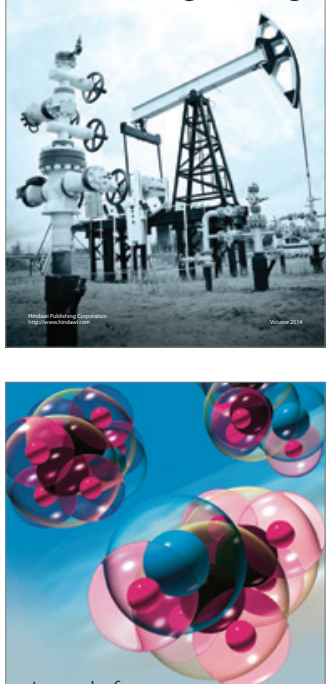

Fuels
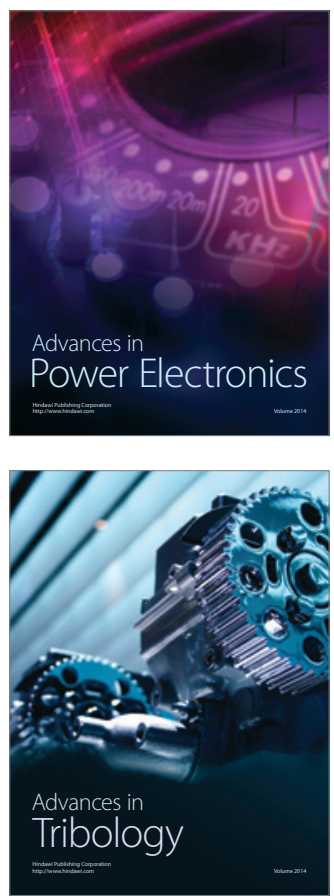

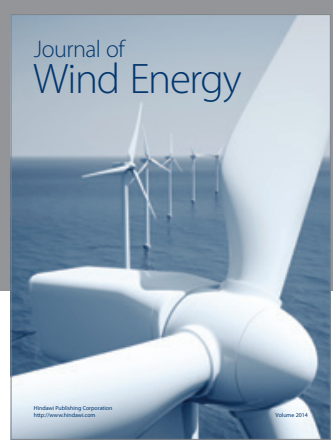

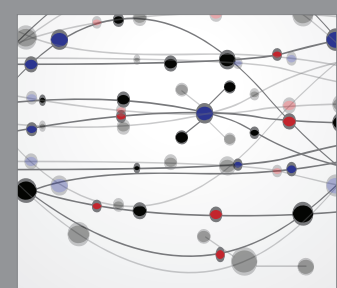

The Scientific World Journal

Submit your manuscripts at http://www.hindawi.com

Journal of

Structures
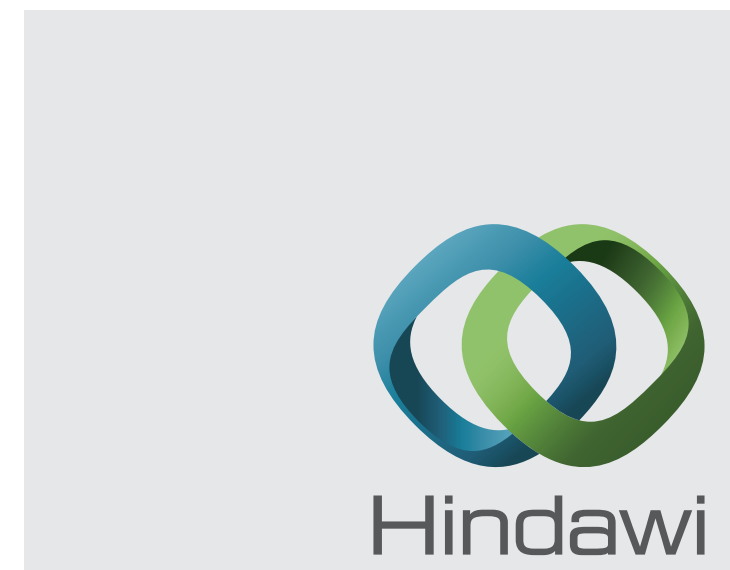

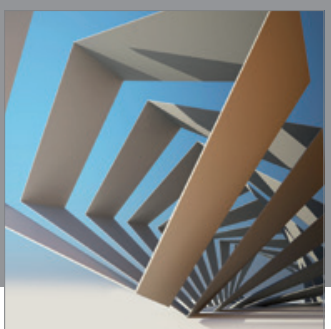

Rotating

Machinery
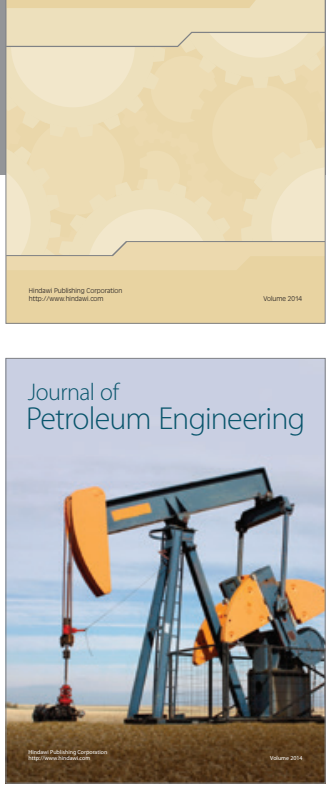

Journal of

Solar Energy
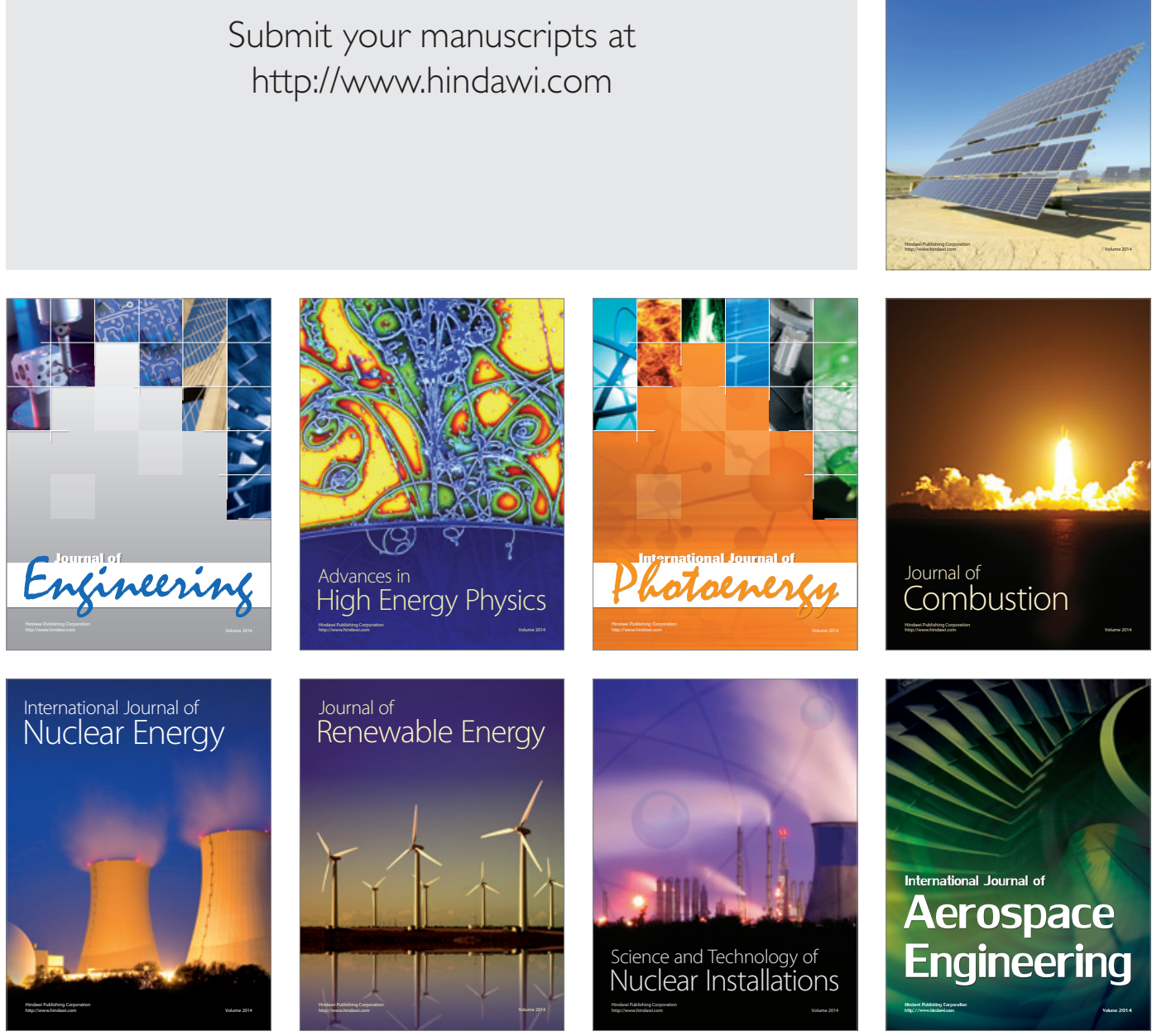